\title{
Propagators of light scalar mesons
}

\author{
N.N. Achasov ${ }^{*}$ and A.V. Kiselev ${ }^{\dagger}$ \\ Laboratory of Theoretical Physics, Sobolev Institute for Mathematics, Novosibirsk, 630090, Russia
}

(November 3, 2018)

\begin{abstract}
For the first time, as far as we know, in field theory are found explicit forms of propagators satisfying the Källen - Lehmann representation. To be exact, it is shown that scalar meson propagators, taking into account a virtual intermediate state contribution to the resonance self-energy, satisfy the Källen - Lehmann representation in the wide domain of coupling constants of the light scalar mesons with the two-particle states. It is proposed to use these propagators in routine fitting data about light scalar mesons to reveal physics underlying the light scalar mesons.
\end{abstract}

PACS number(s): 11.10.St, 11.55.Fv, 12.39.Fe

*achasov@math.nsc.ru

${ }^{\dagger}$ kiselev@math.nsc.ru 
Study of the nature of light scalar resonances has become a central problem of nonperturbative QCD. The point is that the elucidation of their nature is important for understanding both the confinement physics and the chiral symmetry realization way in the low energy region, i.e., the main consequences of QCD in the hadron world. Actually, what kind of interaction at low energy is the result of the confinement in the chiral limit? Is QCD equivalent to the nonlinear $\sigma$ model or the linear one at low energy?

The experimental nonet of the light scalar mesons [1], the putative $f_{0}(600)$ (or $\sigma(600)$ ) and $\kappa(700-900)$ mesons and the well established $f_{0}(980)$ and $a_{0}(980)$ mesons [2], suggests the $U_{L}(3) \times U_{R}(3)$ linear $\sigma$ model. History of the linear $\sigma$ model is rather long, so that the list of its participants, quoted in Ref. [3], is far from complete. Hunting the light $\sigma$ and $\kappa$ mesons had begun in the sixties already and a preliminary information on the light scalar mesons in Particle Data Group Reviews had appeared at that time. But long-standing unsuccessful attempts to prove their existence in a conclusive way entailed general disappointment and information on these states disappeared from Particle Data Group Reviews. One of principal reasons against the $\sigma$ and $\kappa$ mesons was the fact that both $\pi \pi$ and $\pi \kappa$ scattering phase shifts do not pass over $90^{\circ}$ at putative resonance masses. Situation changes when it was shown [4] that in the linear $\sigma$ model there is a negative background phase which hides the $\sigma$ meson. It has been made clear that shielding of wide lightest scalar mesons in chiral dynamics is very natural. This idea was picked up, see, for example, Ref. [5], and triggered new wave of theoretical and experimental searches for the $\sigma$ and $\kappa$ mesons, see Particle Data Group Review [1].

In theory the principal problem is impossibility to use the linear $\sigma$ model in the tree level approximation inserting widths into $\sigma$ meson propagators because such an approach breaks the both unitarity and Adler self-consistency conditions [4]. Strictly speaking, the comparison with the experiment requires the nonperturbative calculation of the process amplitudes [6]. Nevertheless, now there are the possibilities to estimate odds of the $U_{L}(3) \times$ $U_{R}(3)$ linear $\sigma$ model to the underlying physics of the light scalar mesons in phenomenology [7]. Really, even now there is a huge body of information about the $S$ waves of different two-particle pseudoscalar states and what is more the relevant information goes to press almost continuously from BES, BNL, CERN, CESR, DA $\Phi$ NE, FNAL, KEK, SLAC, and others. As for theory, we know quite a lot about the scenario under discussion: the nine scalar mesons, the putative chiral masking [4] of the $\sigma(600)$ and $\kappa(700-900)$ mesons, the unitarity and Adler self-consistency conditions. In addition, there is the light scalar meson treatment motivated by field theory. The foundations of this approach were formulated in Refs. [8-11]. In Refs. [8-10] there were introduced into practice the propagators of light scalar mesons $1 / D_{R}\left(m^{2}\right)[12]$. The inverse propagator

$$
D_{R}\left(m^{2}\right)=m_{R}^{2}-m^{2}+R e\left(\Pi_{R}\left(m_{R}^{2}\right)\right)-\Pi_{R}\left(m^{2}\right)
$$

where $R e\left(\Pi_{R}\left(m_{R}^{2}\right)\right)-\Pi_{R}\left(m^{2}\right)$ takes into account the finite width corrections of the light scalar $R$ resonance, which take into account the contribution of the two-particle virtual intermediate $a b$ states to self-energy of the $R$ resonance,

$$
\Pi_{R}\left(m^{2}\right)=\sum_{a b} \Pi_{R}^{a b}\left(m^{2}\right) .
$$

In real axis of $m^{2}$ 


$$
\operatorname{Im}\left(\Pi_{R}\left(m^{2}\right)\right)=m \Gamma_{R}(m)=m \sum_{a b} \Gamma(R \rightarrow a b, m) \theta\left(m-m_{a}-m_{b}\right)
$$

where

$$
\Gamma(R \rightarrow a b, m)=\frac{g_{R a b}^{2}}{16 \pi m} \rho_{a b}\left(m^{2}\right)
$$

is the width of the $R \rightarrow a b$ decay, $m=m_{a b}$ is the invariant mass of the $a b$ state, $g_{R a b}$ is the coupling constant of the $R$ scalar resonance with the two particle $a b$ state [13], and

$$
\rho_{a b}\left(m^{2}\right)=\sqrt{\left(1-\frac{m_{+}^{2}}{m^{2}}\right)\left(1-\frac{m_{-}^{2}}{m^{2}}\right)}, \quad m_{ \pm}=m_{a} \pm m_{b} .
$$

Below is shown that propagators under discussion satisfy the Källen - Lehmann representation [14]

$$
\frac{1}{D_{R}\left(m^{2}\right)}=\frac{1}{\pi} \int_{m_{0}^{2}}^{\infty} \frac{\operatorname{Im}\left(\frac{1}{D_{R}\left(\bar{m}^{2}\right)}\right)}{\bar{m}^{2}-m^{2}-i \varepsilon} d \bar{m}^{2}=\frac{1}{\pi} \int_{m_{0}^{2}}^{\infty} \frac{\bar{m} \Gamma_{R}(\bar{m})}{\left|D_{R}\left(\bar{m}^{2}\right)\right|^{2}\left(\bar{m}^{2}-m^{2}-i \varepsilon\right)} d \bar{m}^{2}
$$

in the wide domain of coupling constants of the scalar $R$ resonance with the two-particle $a b$ states, here $m_{0}^{2}=\left(m_{a}+m_{b}\right)^{2}$ is the lowest threshold.

Recall that the one-loop contribution to the self-energy of the $R$ resonance from the twoparticle intermediate $a b$ states satisfies the dispersion relation with one subtraction. Let us subtract at $m^{2}=\left(m_{a}+m_{b}\right)^{2}[15]$

$$
\Pi_{R}^{a b}\left(m^{2}\right)=\frac{1}{\pi}\left[m^{2}-\left(m_{a}+m_{b}\right)^{2}\right] \int_{\left(m_{a}+m_{b}\right)^{2}}^{\infty} \frac{\bar{m} \Gamma(R \rightarrow a b, \bar{m})}{\left[\bar{m}^{2}-\left(m_{a}+m_{b}\right)^{2}\right]\left(\bar{m}^{2}-m^{2}-i \varepsilon\right)} d \bar{m}^{2} .
$$

When $m^{2}<\left(m_{a}+m_{b}\right)^{2}$

$$
\Pi_{R}^{a b}\left(m^{2}\right)<0, \quad \frac{d \Pi_{R}^{a b}\left(m^{2}\right)}{d m^{2}}=\frac{1}{\pi} \int_{\left(m_{a}+m_{b}\right)^{2}}^{\infty} \frac{\bar{m} \Gamma(R \rightarrow a b, \bar{m})}{\left(\bar{m}^{2}-m^{2}\right)^{2}} d \bar{m}^{2}>0 .
$$

Clearly, the Källen - Lehmann representation takes place only if

$$
D_{R}(z)=m_{R}^{2}-z+R e\left(\Pi_{R}\left(m_{R}^{2}\right)\right)-\Pi_{R}(z) \neq 0
$$

over the whole complex plane $m^{2} \equiv z=x+i y$.

Let us consider at first when $\operatorname{Im}\left(D_{R}\left(x=m^{2}\right)\right)=0$. It follows from Eqs. (1), (2), and (7) that

$$
\operatorname{Im}\left(D_{R}(z)\right)=-y\left(1+\sum_{a b} \frac{1}{\pi} \int_{\left(m_{a}+m_{b}\right)^{2}}^{\infty} \frac{\bar{m} \Gamma(R \rightarrow a b, \bar{m})}{\left|\bar{m}^{2}-z\right|^{2}} d \bar{m}^{2}\right) .
$$

Consequently, $\operatorname{Im}\left(D_{R}(z)\right)=0$ only in real axis, when $y=0$. In additional, see Eqs. (1) and $(3), \operatorname{Im}\left(D_{R}\left(x=m^{2}\right)\right)=-m \Gamma_{R}(m)$. So, $\operatorname{Im}\left(D_{R}\left(x=m^{2}\right)\right)=0$ only at $m^{2}<m_{0}^{2}$.

Let us find now noughts of $\operatorname{Re}\left(D_{R}\left(x=m^{2}\right)\right)=D_{R}\left(x=m^{2}\right)$ at $m^{2}<m_{0}^{2}$ 


$$
D_{R}\left(m^{2}\right)=m_{R}^{2}-m^{2}+R e\left(\Pi_{R}\left(m_{R}^{2}\right)\right)-\Pi_{R}\left(m^{2}\right)=0 .
$$

The inverse propagator $D_{R}\left(m^{2}\right)$ increases monotone when $m^{2}$ decreases at $m^{2}<m_{0}^{2}$ because

$$
\frac{d D_{R}\left(m^{2}\right)}{d m^{2}}=-1-\frac{d \Pi_{R}\left(m^{2}\right)}{d m^{2}}=-1-\frac{1}{\pi} \int_{m_{0}^{2}}^{\infty} \frac{\bar{m} \Gamma_{R}(\bar{m})}{\left(\bar{m}^{2}-m^{2}\right)^{2}} d \bar{m}^{2}<0 .
$$

So, the single nought of $D_{R}\left(m^{2}\right)$ is provided when

$$
D_{R}\left(m_{0}^{2}\right)=m_{R}^{2}-m_{0}^{2}+R e\left(\Pi_{R}\left(m_{R}^{2}\right)\right)-\Pi_{R}\left(m_{0}^{2}\right) \leq 0
$$

or

$$
m_{R}^{2}-m_{0}^{2} \leq \Pi_{R}\left(m_{0}^{2}\right)-R e\left(\Pi_{R}\left(m_{R}^{2}\right)\right)=\sum_{a b} \Pi_{R}^{a b}\left(m_{0}^{2}\right)-\sum_{a b} R e\left(\Pi_{R}^{a b}\left(m_{R}^{2}\right)\right)
$$

The left-hand side of Eq. (14) is positive. As for the right-hand side of Eq. (14), the contribution of the every $a b$ channel, under the threshold of which the $R$ resonance is, $m_{R}<m_{a}+m_{b}$,

$$
\Pi_{R}^{a b}\left(m_{0}^{2}\right)-R e\left(\Pi_{R}^{a b}\left(m_{R}^{2}\right)\right)=\Pi_{R}^{a b}\left(m_{0}^{2}\right)-\Pi_{R}^{a b}\left(m_{R}^{2}\right)<0
$$

according to Eqs. (8). So, only the $a b$ channels, the thresholds of which are under the $R$ resonance, $m_{a}+m_{b}<m_{R}$, can bring the threat to the Källen - Lehmann representation.

As for the light scalar meson case, $\sigma(600), \kappa(700-900), f_{0}(980), a_{0}(980)$, there is only one channel, the threshold of which is under the $R$ resonance, $\pi \pi, \pi K, \pi \pi, \pi \eta$ respectively. Let us consider at first the one-channel case when the decay threshold is lower than the $R$ resonance, $m_{a}+m_{b}<m_{R}$. This scenario is most vulnerable from the point of the Källen Lehmann representation view. One has

$$
\begin{aligned}
& \Pi_{R}\left(m^{2}\right)=\Pi_{R}^{a b}\left(m^{2}\right) \equiv \frac{g_{R a b}^{2}}{16 \pi^{2}} P^{a b}\left(m^{2}\right)=\frac{g_{R a b}^{2}}{16 \pi^{2}}\left\{\frac{\left(m^{2}-m_{+}^{2}\right)}{m^{2}} \frac{m_{-}}{m_{+}} \ln \frac{m_{a}}{m_{b}}\right. \\
& \left.+\rho_{a b}\left(m^{2}\right)\left[i \pi+\ln \frac{\sqrt{m^{2}-m_{-}^{2}}-\sqrt{m^{2}-m_{+}^{2}}}{\sqrt{m^{2}-m_{-}^{2}}+\sqrt{m^{2}-m_{+}^{2}}}\right]\right\}
\end{aligned}
$$

at $m \geq m_{+}=m_{a}+m_{b}$, here and hereafter $m_{a} \geq m_{b}$. As is evident from Eqs. (14) and $\Pi_{R}^{a b}\left(m_{0}^{2}=m_{+}^{2}\right)=0$, the Källen - Lehmann representation is valid if

$$
m_{R}^{2}-m_{+}^{2}>-\frac{g_{R a b}^{2}}{16 \pi^{2}} R e\left(P^{a b}\left(m_{R}^{2}\right)\right)
$$

The one-channel propagators can be actual when treating the $\sigma(600)$ resonance, the $\pi \pi$ channel, and the $\kappa(700-900)$ resonance, the $\pi K$ channel. Let us find what the coupling constants are allowed from the Källen - Lehmann representation view in these cases. 


$$
\begin{aligned}
& g_{\sigma \pi \pi}^{2} / 16 \pi^{2}<\left(g_{\sigma \pi \pi}^{c}\right)^{2} / 16 \pi^{2}=-\left(m_{\sigma}^{2}-4 m_{\pi}^{2}\right) / \operatorname{Re}\left(P^{\pi \pi}\left(m_{\sigma}^{2}\right)\right) \approx 0.1 \mathrm{GeV}^{2} \\
& g_{\kappa \pi K}^{2} / 16 \pi^{2}<\left(g_{\kappa \pi K}^{c}\right)^{2} / 16 \pi^{2}=-\left[m_{\kappa}^{2}-\left(m_{\pi}+m_{K}\right)^{2}\right] / \operatorname{Re}\left(P^{\pi \kappa}\left(m_{\kappa}^{2}\right)\right) \approx 0.4 \mathrm{GeV}^{2}
\end{aligned}
$$

where $g_{\sigma \pi \pi}^{2}=1.5 g_{\sigma \pi^{+} \pi^{-}}^{2}, g_{\kappa \pi K}^{2}=1.5 g_{\kappa^{+} \pi^{+} K^{0}}^{2}, m_{\kappa}=0.8 \mathrm{GeV} . \Gamma\left(\sigma \rightarrow \pi \pi, m_{\sigma}\right)<\Gamma_{\sigma}^{c} \approx 0.5$ $\mathrm{GeV}, \Gamma\left(\kappa \rightarrow \pi K, m_{\kappa}\right)<\Gamma_{\kappa}^{c} \approx 0.8 \mathrm{GeV}$.

The two-channel propagators are often used when treating the $f_{0}(980)$ resonance, the $\pi \pi$ and $K \bar{K}$ channels, and the $a_{0}(980)$ resonance, the $\pi \eta$ and $K \bar{K}$ channels. To consider this issue we need

$$
\Pi_{R}^{a b}\left(m^{2}\right)=\frac{g_{R a b}^{2}}{16 \pi^{2}}\left[\frac{\left(m^{2}-m_{+}^{2}\right)}{m^{2}} \frac{m_{-}}{m_{+}} \ln \frac{m_{a}}{m_{b}}-2\left|\rho_{a b}(m)\right| \arctan \frac{\sqrt{m^{2}-m_{-}^{2}}}{\sqrt{m_{+}^{2}-m^{2}}}\right]
$$

at $m_{-}=m_{a}-m_{b} \leq m \leq m_{+}=m_{a}+m_{b}$.

As is easy to see from Eq. (14), there are the $\left(g_{f_{0} K \bar{K}} / g_{f_{0} \pi \pi}\right)^{2}$ and $\left(g_{a_{0} K \bar{K}} / g_{a_{0} \pi \eta}\right)^{2}$ domains in the two-channel cases where the right sides $\left(R=f_{0}\right.$ or $\left.R=a_{0}\right)$ of Eq. (14) are negative or vanish, in other words, the Källen - Lehmann representation holds for any $g_{f_{0} \pi \pi}^{2}$ and $g_{a_{0} \pi \eta}^{2}$ respectively:

$$
\begin{aligned}
& \left(g_{f_{0} K \bar{K}} / g_{f_{0} \pi \pi}\right)^{2} \geq r_{f_{0}}^{c}=\operatorname{Re}\left(P^{\pi \pi}\left(m_{f_{0}}^{2}\right)\right) /\left[P^{K \bar{K}}\left(4 m_{\pi}^{2}\right)-P^{K \bar{K}}\left(m_{f_{0}}^{2}\right)\right] \approx 2.5 \\
& \left(g_{a_{0} K \bar{K}} / g_{a_{0} \pi \eta}\right)^{2} \geq r_{a_{0}}^{c}=\operatorname{Re}\left(P^{\pi \eta}\left(m_{a_{0}}^{2}\right)\right) /\left[P^{K \bar{K}}\left(\left(m_{\pi}+m_{\eta}\right)^{2}\right)-P^{K \bar{K}}\left(m_{a_{0}}^{2}\right)\right] \approx 0.8
\end{aligned}
$$

where $g_{f_{0} \pi \pi}^{2}=1.5 g_{f_{0} \pi^{+} \pi^{-}}^{2}, g_{f_{0} K \bar{K}}^{2}=2 g_{f_{0} K^{+} K^{-}}^{2}$, and $g_{a_{0} K \bar{K}}^{2}=2 g_{a_{0}^{0} K^{+} K^{-}}^{2}=g_{a_{0}^{+} K^{+} \bar{K}^{0}}^{2}$.

The ratios of the coupling constants, when the $f_{0}(980)$ and $a_{0}(980)$ resonances were treated with the propagators under discussion, satisfied the requirements (20).

As for outside of these domains, the Källen - Lehmann representation allows the following coupling constants :

$$
\begin{aligned}
& \frac{g_{f_{0} \pi \pi}^{2}}{16 \pi^{2}}<\frac{\left(g_{f_{0} \pi \pi}^{c}\right)^{2}}{16 \pi^{2}}=\left(m_{f_{0}}^{2}-4 m_{\pi}^{2}\right) /\left\{-\operatorname{Re}\left(P^{\pi \pi}\left(m_{f_{0}}^{2}\right)\right)\right. \\
& \left.+\left(g_{f_{0} K \bar{K}} / g_{f_{0} \pi \pi}\right)^{2}\left[P^{K \bar{K}}\left(4 m_{\pi}^{2}\right)-P^{K \bar{K}}\left(m_{f_{0}}^{2}\right)\right]\right\} \\
& \frac{g_{a_{0} \pi \eta}^{2}}{16 \pi^{2}}<\frac{\left(g_{a_{0} \pi \eta}^{c}\right)^{2}}{16 \pi^{2}}=\left[m_{a_{0}}^{2}-\left(m_{\pi}+m_{\eta}\right)^{2}\right] /\left\{-\operatorname{Re}\left(P^{\pi \eta}\left(m_{a_{0}}^{2}\right)\right)\right. \\
& \left.+\left(g_{a_{0} K \bar{K}} / g_{a_{0} \pi \eta}\right)^{2}\left[P^{K \bar{K}}\left(\left(m_{\pi}+m_{\eta}\right)^{2}\right)-P^{K \bar{K}}\left(m_{a_{0}}^{2}\right)\right]\right\} .
\end{aligned}
$$

To experience what values of $g_{f_{0} \pi \pi}$ and $g_{a_{0} \pi \eta}$ are allowed by the Källen - Lehmann representation when Eqs. (20) are not satisfied, we consider a deliberately non-real case of the weak coupling of $f_{0}(980)$ and $a_{0}(980)$ with the $K \bar{K}$ channel which gives a very conservative estimate. Suppose $\left(g_{f_{0} K \bar{K}} / g_{f_{0} \pi \pi}\right)^{2}=\left(g_{a_{0} K \bar{K}} / g_{a_{0} \pi \eta}\right)^{2}=1 / 3$ then 
$\left(g_{f_{0} \pi \pi}\right)^{2} / 16 \pi^{2}<\left(g_{f_{0} \pi \pi}^{c}\right)^{2} / 16 \pi^{2} \approx 0.3 \mathrm{GeV}^{2}, \Gamma\left(f_{0} \rightarrow \pi \pi, m_{f_{0}}\right)<\Gamma_{f_{0}}^{c} \approx 0.9 \mathrm{GeV}$, and $\left(g_{a_{0} \pi \eta}\right)^{2} / 16 \pi^{2}<\left(g_{a_{0} \pi \eta}^{c}\right)^{2} / 16 \pi^{2} \approx 0.9 \mathrm{GeV}^{2}, \Gamma\left(a_{0} \rightarrow \pi \eta, m_{a_{0}}\right)<\Gamma_{a_{0}}^{c} \approx 1.8 \mathrm{GeV}[16]$.

Taking into account the $\eta \eta, \eta \eta^{\prime}$ and $\pi \eta^{\prime}$ channels only extends the coupling constant domains where the Källen - Lehmann representation holds. The inclusion of the $\eta \eta^{\prime}$ and $\pi \eta^{\prime}$ channels requires

$$
\Pi_{R}^{a b}\left(m^{2}\right)=\frac{g_{R a b}^{2}}{16 \pi^{2}}\left[\frac{\left(m^{2}-m_{+}^{2}\right)}{m^{2}} \frac{m_{-}}{m_{+}} \ln \frac{m_{a}}{m_{b}}-\rho_{a b}(m) \ln \frac{\sqrt{m_{+}^{2}-m^{2}}-\sqrt{m_{-}^{2}-m^{2}}}{\sqrt{m_{+}^{2}-m^{2}}+\sqrt{m_{-}^{2}-m^{2}}}\right]
$$

at $m \leq m_{-}=m_{a}-m_{b}$ and $m^{2} \leq 0$.

The Källen - Lehmann representation guarantees the unitarity condition for the branching ratious

$$
1 \equiv \sum_{a b} \operatorname{Br}(R \rightarrow a b)=\sum_{a b} \frac{1}{\pi} \int_{\left(m_{a}+m_{b}\right)^{2}}^{\infty} \frac{\bar{m} \Gamma(R \rightarrow a b, \bar{m})}{\left|D_{R}\left(\bar{m}^{2}\right)\right|} d \bar{m}^{2}
$$

which follows from Eqs. (6), (1), and (16) when $m^{2} \rightarrow \infty$ [17].

Recall that to satisfy Eq. (23) the very popular Flatté formulas [18] require a factor which considerably differs from 1 , see, for example, [19]. The Flatté formulas ensue from our ones by the following substitutions

$$
\begin{aligned}
& R e\left(\Pi_{R}^{a b}\left(m_{R}^{2}\right)\right)-\Pi_{R}^{a b}\left(m^{2}\right) \rightarrow-i \frac{g_{R a b}^{2}}{16 \pi} \rho_{a b}\left(m^{2}\right), \quad m_{a}+m_{b} \leq m ; \\
& R e\left(\Pi_{R}^{a b}\left(m_{R}^{2}\right)\right)-\Pi_{R}^{a b}\left(m^{2}\right) \rightarrow \frac{g_{R a b}^{2}}{16 \pi}\left|\rho_{a b}\left(m^{2}\right)\right|, \quad m_{a}-m_{b} \leq m \leq m_{a}+m_{b} .
\end{aligned}
$$

As for $m \leq m_{a}-m_{b}$ and $m^{2}<0$, the analytic continuation of the Flatté formulas in this region has no physical sense. In addition, the Flatté formulas keep back traps for users. The point is that $R e\left(D_{R}\left(m^{2}\right)\right)$ has nought not at $m_{R}^{2}$ but at the renormalized mass square $M_{R}^{2}=m_{R}^{2}+\left(g_{R K \bar{K}}^{2} / 16 \pi\right)\left|\rho_{K \bar{K}}\left(M_{R}^{2}\right)\right|$ in the two channel $f_{0}(980)$ and $a_{0}(980)$ treatment with the Flatté formulas. Consequently, as long as a user would like to associate the peak location in the mass distribution

$$
\frac{d N_{a b}(m)}{d m}=N_{a b} \frac{2 m^{2}}{\pi} \frac{\Gamma(R \rightarrow a b, m)}{\left|D_{R}\left(m^{2}\right)\right|^{2}}, \quad R=f_{0}(980), a b=\pi \pi ; \quad R=a_{0}(980), a b=\pi \eta
$$

with $m_{R}^{2}$, he will be in the region of the weak coupling of $f_{0}(980)$ and $a_{0}(980)$ with the $K \bar{K}$ channel, a more detailed consideration can be found in Ref. [20].

The propagators under discussion are used routinely by the Sobolev Institute for Mathematics Group [21]. They are used also by the SND [22], CMD-2 [23], and KLOE [24] Collaborations in treating the $\phi \rightarrow \gamma f_{0}(980) \rightarrow \gamma \pi \pi$ and $\phi \rightarrow \gamma a_{0}(980) \rightarrow \gamma \pi \eta$ decays. In addition, these propagators are used also in Refs. [25]. They have the ideal properties, being only a little more complicated than the Flatté formulas [26]. We propose to use these propagators routinely in treating the mass spectra $f_{0}(980) \rightarrow \pi \pi$ and $a_{0}(980) \rightarrow \pi \eta$ to reveal physics underlying the light scalar mesons.

Really, all information (coupling constants and masses) on scalar mesons is extracted from spectra, but the study of spectra requires knowledge of propagators. There are two 
means. 1) To use the Flatté formulas, which have Quantum Mechanics origin. These formulas mean that virtual particles do not contribute to a resonance self-energy, that is, these formulas mean that a resonance is a weakly bound system. 2) To use our formulas, which have Quantum Field Theory origin and take into account the contribution of virtual particles to a resonance self-energy. Our formulas are adequate to description of compact states $\left(q \bar{q}, q^{2} \bar{q}^{2}\right.$, and so on) strongly coupled with decay channels. In addition, our formulas pass into the Flatté ones at the limit of weak coupling.

This work was supported in part by the RFBR Grant No. 02-02-16061 and the Presidential Grant No. 2339.2003.2 for support of Leading Scientific Schools. A.V. Kiselev also thanks very much Dynasty Foundation and ICFPM for scholarship. 


\section{REFERENCES}

[1] Particle Data Group-2002, K. Hagiwara et al., Phys. Rev. D 66010001 (2002).

[2] The nontrivial nature of the well established light scalar resonances $f_{0}(980)$ and $a_{0}(980)$ is no longer denied practically anybody. In particular, there exist numerous evidences in favour of the four-quark $\left(q^{2} \bar{q}^{2}\right)$ structure of these states, see, for example, N.N. Achasov, Nucl. Phys. A 728, 425 (2003), Yad.Fiz. 65, 573 (2002) [Phys. At. Nucl. 65, 546 (2002)], and references therein. As for the nonet as a whole, a look at Particle Data Group Review [1] gives an idea of the four-quark structure of the light scalar meson nonet, $f_{0}(600)$ (or $\left.\sigma\right), \kappa(700-900), f_{0}(980)$, and $a_{0}(980)$, inverted in comparison with the classical two-quark $(q \bar{q})$ vector meson nonet. Really, while such a nonet cannot be treated as the $q \bar{q}$ one in the naive quark model, it can be easily understood as the $q^{2} \bar{q}^{2}$ nonet as shown in R.L. Jaffe, Phys. Rev. D 15, 267, 281 (1977); D. Black, A.Fariborz, F. Sannino, and J. Schechter, Phys.Rev. D 59, 074026 (1999); M. Harada, F. Sannino, and J. Schechter, Phys. Rev. D 69, 034005 (2004).

[3] M. Gell-Mann and M. Levy, Nuovo Cimento 16, 705 (1960);

S. Weinberg, Phys. Rev. Lett. 18, 188 (1967);

B.W. Lee, Nucl. Phys. B9, 649 (1969);

S. Gasiorowicz and D.A. Geffen, Rev. Mod. Phys. 41, 531 (1969);

J. Schechter and Y. Ueda, Phys. Rev. D 3, 168, 2874 (1971);

C. Rosenzweig, J. Schechter, and C.G. Trahern, Phys. Rev. D 21, 3388 (1980);

P. Di Vecchia and G. Veneziano, Nucl. Phys. B 171, 253 (1980);

G.'t Hooft, hep-th/9903189.

[4] N.N. Achasov and G.N. Shestakov, Phys. Rev. D 49, 5779 (1994).

[5] M. Ishida, S. Ishida, and T. Ishida, Prog.Theor.Phys. 99, 1031 (1998).

[6] A few approximate non-perturbative solutions in the frame of the simplest linear $\sigma$ model are found in Ref. [4].

[7] To be on the safe side, notice that the linear $\sigma$ model does not contradict to non- $q \bar{q}$ nature of the low lying scalars because Quantum Fields can contain different virtual particles in different regions of virtuality.

[8] N.N. Achasov, S.A. Devyanin, and G.N. Shestakov, Phys. Lett. 88B, 367 (1979).

[9] N.N. Achasov, S.A. Devyanin, and G.N. Shestakov, Phys. Lett. 96B, 168 (1980).

[10] N.N. Achasov, S.A. Devyanin, and G.N. Shestakov, Yad. Fiz. 32, 1098 (1980)[Sov. J. Nucl. Phys. 32, 566 (1980)].

[11] N.N. Achasov, S.A. Devyanin and G.N. Shestakov, Z. Phys. C 22, 53 (1984);

Usp. Fiz. Nauk. 142, 361 (1984) [Sov. Phys. Usp. 27, 161 (1984)].

[12] This approach gave indirect evidences of the four-quark nature of the $f_{0}(980)$ and $a_{0}(980)$ mesons $[9-11]$.

[13] One can introduce the renormalized coupling constants $\bar{g}_{R a b}^{2}=g_{R a b}^{2} /[1+$ $\left.d \Pi_{R}\left(m^{2}\right) /\left.d m^{2}\right|_{m=m_{R}}\right]$, expanding $\operatorname{Re}\left(\Pi_{R}\left(m_{R}^{2}\right)\right)-\Pi_{R}\left(m^{2}\right)=-d \Pi_{R}\left(m^{2}\right) /\left.d m^{2}\right|_{m=m_{R}}$ $\times\left(m^{2}-m_{R}^{2}\right)+\ldots$, but the bare coupling constants $g_{R a b}$ are more informative for clearing up the resonance nature. In addition, one cannot take into account only the first term in the above expansion even in the resonance region when the matter concerns the $a_{0}(980)$ and $f_{0}(980)$ resonances because these states are close to the $K \bar{K}$ channel, see Refs. [10] and N.N. Achasov, S.A. Devyanin, and G.N. Shestakov, Phys. Lett. 102B, 196 (1981). 
[14] The $a$ and $b$ states are the stable pseudoscalar ones in all specific cases which we consider, but all formulas are correct also when the $a$ and $b$ states are the stable scalar ones. If one considers the above $R$-resonance self-energy as the one chiral loop approximation in the linear $\sigma$ model, one would expect that the higher order corrections reduce basically to the coupling constant and mass renormalization. We mention that the preliminary estimation in the two chiral loop approximation supports these expectations. In other words, one would expect that the formula in the one chiral loop approximation with the physical coupling constants and mass is close to the exact formula.

[15] Eq. (1) does not depend on the subtraction of $\Pi_{R}^{a b}\left(m^{2}\right)$ at any $m^{2} \leq\left(m_{a}+m_{b}\right)^{2}$, or more generally, does not depend on an addition of any real constant to $\Pi_{R}^{a b}\left(m^{2}\right)$.

[16] The values $\Gamma\left(f_{0} \rightarrow \pi \pi, m_{f_{0}}\right)$ and $\Gamma\left(a_{0} \rightarrow \pi \eta, m_{a_{0}}\right)$ characterize the visible widths of $f_{0}(980)$ and $a_{0}(980)$ when they couple weakly to the $K \bar{K}$ channel.

[17] Eq. (23) for $f_{0} \rightarrow \pi \pi, K \bar{K}$ and $a_{0} \rightarrow \pi \eta, K \bar{K}$ was checked numerically with a high accuracy in Ref. [10], but the general proof was absent.

[18] S. Flatte', Phys. Lett. 63B, 224 (1976).

[19] L.A. Kondratyuk et al., Yad. Fiz. 66, 155 (2003) [Phys. Atom. Nucl. 66, 152 (2003)].

[20] N.N. Achasov and V.V.Gubin, Phys. Lett. B 363, 106 (1995); Phys. Rev. D 56, 4084 (1997); N.N. Achasov, V.V. Gubin, and V.I. Shevchenko, Phys. Rev. D 56, 203 (1997).

[21] In addition to the papers cited above, see N.N. Achasov and V.N. Ivanchenko, Nucl. Phys. B 315, 465 (1989), N.N. Achasov and V.V. Gubin, Phys. Rev. D 56, 4084 (1997), Phys. Rev. D 63, 094007 (2001), N.N. Achasov and A.V. Kiselev, Phys. Rev. D 68, 014006 (2003) and others.

[22] M.N. Achasov et al., Phys. Lett. B 438, 441 (1998); Phys. Lett. B 440, 442 (1998); Phys. Lett. B 479, 53 (2000); Phys. Lett. B 485, 349 (2000).

[23] R.R. Akhmetshin et al., Phys. Lett. B 462, 380 (1999).

[24] A. Aloisio et al., Phys.Lett. B 536, 209 (2002); Phys.Lett. B 537, 21 (2002).

[25] A. Bramon et al., Eur. Phys. J. C 26, 253 (2002); B. Kerbikov, Phys. Lett. B 596, 200 (2004).

[26] The explicit analytic form of the new large family of the very complicated integrals (6) is found in our consideration as a by-product. 